\title{
ОПРЕДЕЛЕНИЕ ПОЛЕЙ НАПРЯЖЕНИЙ ОТ ДЕЙСТВИЯ РАСПРЕДЕЛЕННОЙ НАГРУЗКИ ПРИ НАЛИЧИИ У ПОВЕРХНОСТИ КЛИНОВИДНОГО ДВОЙНИКА
}

\author{
Н. М. Симанович ${ }^{1}$, О. М. Остриков², М. П. Кульгейко \\ ${ }^{1}$ Аспирант кафредры «Механика» учреждения образования «Гомельский государственный \\ технический университет имени П. О. Сухого», Гомель, Беларусь, e-mail: nataliasimoniha1991@gmail.com \\ ${ }^{2}$ К. фриз.-мат. н., доцент, доцент кафедры «Механика» учреждения образования \\ «Гомельский государственный технический университет имени П. О. Сухого», Гомель, Беларусь, е-mail: omostrikov@mail.ru \\ ${ }^{3}$ K. т. н., доцент, доцент кафредры «Графика» учреждения образования \\ «Белорусский государственный университет транспорта», Гомель, Беларусь, e-mail: nataliasimoniha1991@gmail.com
}

\begin{abstract}
Ресрерат
Установлено влияние внешнего десормационного воздействия распределенной нагрузкой на напряжённое состояние в области клиновидного двойника. Показано изменение полей напряжений в приповерхностной области, содержащей двойник, в зависимости от интенсивности, равномерности и величины зоны внешнего силового нагружения.
\end{abstract}

Ключевые слова: механическое двойникование, поля напряжений, распределенная нагрузка.

\section{DETERMINATION OF STRESS FIELDS FROM THE ACTION OF A DISTRIBUTED LOAD IN THE PRESENCE OF A WEDGE-SHAPED TWIN AT THE SURFACE}

\section{Abstract}

\section{N. M. Simanovich, O. M. Ostrikov, M. P. Kulgeiko}

The influence of the external deformation effect of the distributed load on the stress state in the region of the wedge-shaped twin has been established. The change in stress fields in the near-surface region containing a twin is shown, depending on the intensity, uniformity and size of the external force loading zone.

Keywords: mechanical twinning, stress fields, distributed load.

\section{Введение}

Двойникование, наряду со скольжением, является одним из распространенных видов пластической деформации металлов [1-3], а в определенных условиях может быть основным деформационным механизмом [4]. Этот процесс сопровождается концентрацией напряжений в вершинах и на границах двойниковых дефектов. Повышение напряженного состояния в области двойников обуславливает образование микротрещин с возможностью последующего усталостного разрушения материала.

В связи со значительной неопределенностью роли двойникования в деформировании и разрушении металлов и сплавов [3-6] важной задачей является изучение закономерностей и особенностей формирования полей напряжений у границ двойников в условиях воздействия различных факторов десрормационного нагружения. Результат влияния двойникования на состояние и поведение материала определяется рядом условий и факторов, в том числе состоянием поверхности твердого тела.

\section{Постановка задачи}

Процесс разрушения материала характеризуется стадией постоянного накопления повреждений, в результате которого образуются микротрещины [4]. Активную роль в зарождении и развитии микротрещин играет механическое двойникование, которое создаёт дополнительные внутренние напряжения наряду с напряжениями от действия внешней механической нагрузки на поверхности твёрдого тела. Действие внешней нагрузки при наличии двойника создаёт в материале сложное напряжённое состояние, которое определяется условиями внешнего нагружения [7].

Влияние клиновидных двойников на напряжённое состояние материала, находящегося под действием внешней нагрузки, особенно важно на уровне малых объёмов. В этих условиях решение практических задач механики деформируемого твёрдого тела в значительной степени определяется не только величиной внешнего воздействия, но и характером распределения нагружения на поверхности материала. Аналогичные условия могут иметь место, например, при работе электрических контактов [8]. Так наиболее сложной проблемой при конструировании разъёмных контактов является определение оптимальной величины контактного давления. А надёжность их работы определяется как эксплуатационными фракторами (нагрузкой), так и конструктивно-технологическими, в том числе геометрией контактирующей поверхности. То есть работоспособность и надёжность контактов зависит от фактической площади контакта и площади контакта, проводящей ток (рабочей площади). Кроме того, многочисленные удары приводят к пластической деформации и сдвигу поверхностных слоёв. В этих условиях важное значение имеет возможность прогнозирования напряжённого состояния приповерхностной области и поверхностного слоя материала, так как процесс разрушения начинается чаще на поверхности твёрдого тела.

Целью настоящей работы является оценка напряжённого состояния при деформировании поверхности рассредоточенной нагрузкой с наличием в материале клиновидного двойника.

\section{Методика решения задачи}

На рис. 1 схематично представлен клиновидный двойник длиной $L$ и шириной у устья $\mathrm{H}$ с формой границ, описываемой функциями $f_{1}\left(y_{0}\right)$ и $f_{2}\left(y_{0}\right)$. На поверхности $(-m \leq x \leq n)$ в области двойника действует распределённая нагрузка в виде нормального давления $p(x)$. При эквивалентном суммарном давлении $P$ рассмотрим несколько вариантов нагружения: на рис. $1, \mathrm{a}-$ равномерно распределённая нагрузка соответствует условиям плотного равномерного контакта; на рис. 1,б - условиям неплотного (частичного) контакта с неравномерно распределённой нагрузкой. При этом неравномерная нагрузка распределяется в соответствии с выражением

$$
p(s)=\frac{c}{m} s+c \text { или } p(s)=c\left(\frac{s}{m}+1\right),
$$

где $S$ - текущая координата точки пространства вдоль оси OX; C - постоянная, численно равная усилию в точке 0 ; $m$ - начальная граница действия нагрузки.
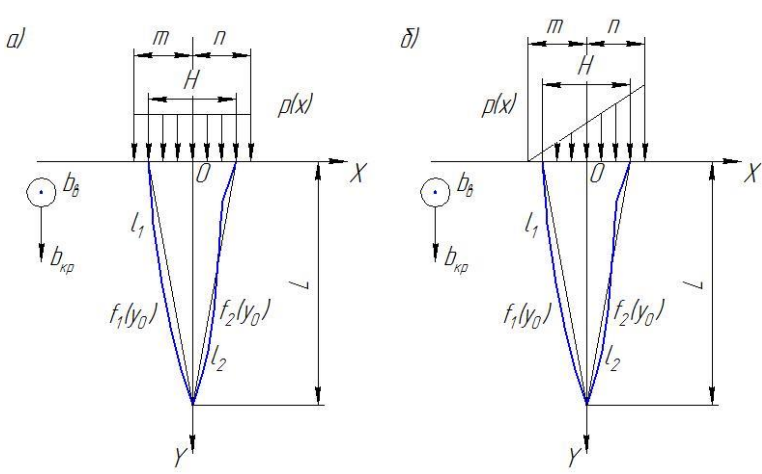

a) равномерной (плотный контакт);

б) неравномерной (частичный контакт)

Рисунок 1 - Схематичное изображение двойника у поверхности с действующей на неё распределённой нагрузкой 
Результирующие напряжения у поверхности, деформированной внешней нагрузкой при наличии двойника, могут быть найдены по формуле [3,7]:

$$
\sigma_{i j}(x, y)=\sigma_{i j}^{\partial \beta}(x, y)+\sigma_{i j}^{p}(x, y),
$$

где $\sigma_{i j}^{\partial \beta}(x, y)$ - напряжения, создаваемые двойником;

$\sigma_{i j}^{p}(x, y)$ - напряжения от внешней нагрузки.

Напряжения, создаваемые рассматриваемым клиновидным двойником, могут быть определены из выражений, представленных B [3,7,9]:

$$
\sigma_{i j}^{\partial B}(x, y)=\sigma_{i j}^{(1)}(x, y)+\sigma_{i j}^{(2)}(x, y),
$$

где $\sigma_{i j}^{(1)}(x, y)$ и $\sigma_{i j}^{(2)}(x, y)$ - напряжения, которые создаются границами двойника.

Как показано в расчетах $[3,7,9]$ :

$$
\begin{aligned}
& \sigma_{i j}^{(1)}(x, y)=\int_{0}^{L} \sqrt{1+\left(f_{1}^{\prime}\left(y_{0}\right)\right)^{2}} \rho_{1}\left(y_{0}\right) \sigma_{i j}^{(1,0)}\left(x, y, y_{0}\right) d y_{0}, \\
& \sigma_{i j}^{(2)}(x, y)=\int_{0}^{L} \sqrt{1+\left(f_{1}^{\prime}\left(y_{0}\right)\right)^{2}} \rho_{2}\left(y_{0}\right) \sigma_{i j}^{(2,0)}\left(x, y, y_{0}\right) d y_{0},
\end{aligned}
$$

где $L$ - длина двойника;

$\rho_{1}\left(y_{0}\right)$ и $\rho_{2}\left(y_{0}\right)$ - плотность двойникующих дислокаций на границах клиновидного двойника;

$\sigma_{i j}^{(1,0)}$ и $\sigma_{i j}^{(2,0)}$ - напряжения, создаваемые на двойниковых границах отдельными дислокациями.

Компоненты напряжений, создаваемых единичной двойникующей дислокацией, находящейся на двойниковой границе при нахождении двойника вблизи поверхности, определяются из соотношений, представленных в [10] по методике [3]. В данной работе, принимая во внимание выводы [11] о том, что наиболее существенное влияние поверхности на конфигурацию полей напряжений оказывается в случае скалывающих напряжений, расчеты выполняются на примере распределения величины сдвиговой компоненты $\sigma_{x y}$ тензора напряжений, то есть определяются напряжения $\sigma_{x y}^{(1,0)} u \sigma_{x y}^{(2,0)}$.

Принимая также допущение, что двойник имеет форму равнобедренного треугольника с прямолинейными границами и плотностью двойникующих дислокаций на них $\rho_{1}=\rho_{2}=\rho=$ const в соответствии с [9], уравнения (4) и (5) примут вид:

$$
\begin{gathered}
\sigma_{x y}^{(1)}=\rho \sqrt{1+\left(\frac{H}{2 L}\right)^{2}} \int_{0}^{L} \sigma_{x y}^{(1,0)}\left(x, y, y_{0}\right) d y_{0} \\
\sigma_{x y}^{(2)}=\rho \sqrt{1+\left(\frac{H}{2 L}\right)^{2}} \int_{0}^{L} \sigma_{x y}^{(2,0)}\left(x, y, y_{0}\right) d y_{0} .
\end{gathered}
$$

Сдвиговые напряжения от действия нормальной распределенной нагрузки $\rho(x)$ определяются в соответствии с рекомендациями $[3,12]$ по формуле:

$$
\sigma_{x y}^{p}=-\frac{2 y^{2}}{\pi} \int_{-m}^{n} \frac{p(s)(x-s) d s}{\left[(x-s)^{2}+y^{2}\right]},
$$

где $m$ и $n$ - границы действия внешней нагрузки; $s$ - параметр интегрирования (см. рис.1).

\section{Результаты и их обсуждение}

Графическая интерпретация результатов представлена на рис. 2-6. В расчётах для $H=10$ мкм и $L=80$ мкм принято [3]: $b_{\kappa p}=0,2 \cdot 10^{-9} \mathrm{M}[13] ; \mu=12,4 \cdot 10^{9}$ Па [1]; $v=0,33$ [13].

Линейная плотность двойникующих дислокаций на двойниковых границах $\rho=0,189$ определена из [3] при межатомном расстоянии $a=3,3 \cdot 10^{-10} \mathrm{M}[14]$.

Для проведения дальнейшего сравнительного анализа по оценке влияния внешнего силового воздействия на напряженное состояние поверхности и в приповерхностной области, содержащей двойник, на рис. 2 представлено распределение сдвиговых напряжений у клиновидного двойника при отсутствии внешней нагрузки.

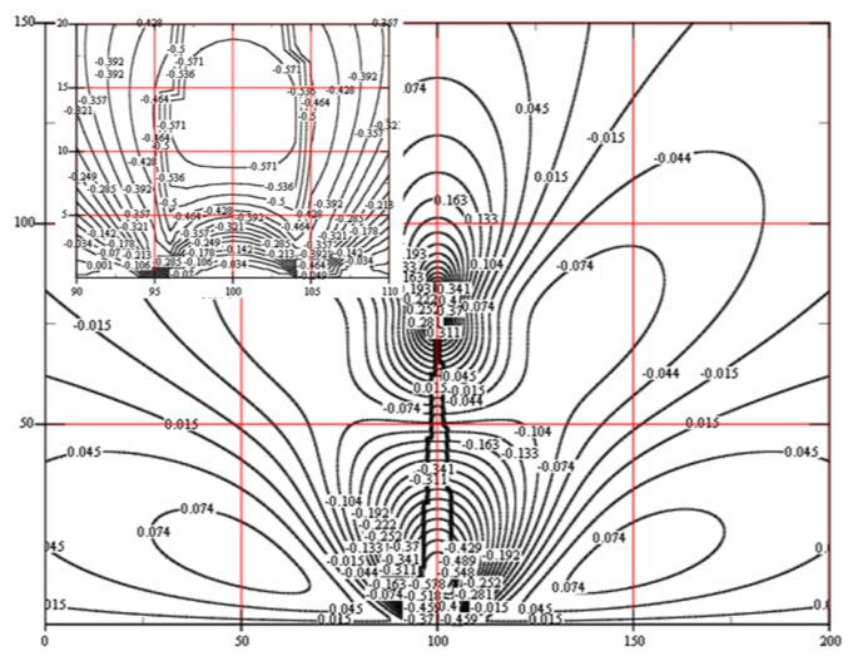

Рисунок 2 - Напряжения у клиновидного двойника при отсутствии внешней нагрузки

В целом конфигурация полей напряжений симметричная относительно плоскости двойникования. Отмечается концентрация напряжений у двойниковых границ и, прежде всего, у вершины двойника. Вдоль двойника от устья к вершине напряжения уменьшаются и приблизительно на расстоянии двух третей длины двойника от поверхности меняют знак, а затем увеличиваются и достигают максимума у вершины двойника. При удалении от устья двойника вдоль поверхности (ось ОХ) напряжения также меняют знак и интенсивно убывают. При этом максимальные отрицательные напряжения наблюдаются внутри двойника на расстоянии 10-15 мкм от поверхности. Это относится и к зонам концентрации положительных напряжений вдали от двойника вдоль оси ОХ, т. е. на поверхности напряжения минимальные, а большие значения имеют место в приповерхностной области.

На рис. 3 представлена картина распределения напряжений $\sigma_{x y}$ при действии на поверхности равномерно распределенной нагрузки $p(s)=3 \mathrm{H} / \mathrm{M}$ в пределах ширины двойника, т. е. при $m=n=H / 2$ (см. рис. 1,а). Здесь имеем несимметричную конфигурацию полей напряжений, особенно у поверхности. На одной границе у устья двойника вблизи поверхности наблюдаются положительные напряжения, у другой - отрицательные, причем численное отношение напряжений по абсолютной величине около трех. Далее по направлению к вершине двойника напряжения вдоль обеих двойниковых границ постепенно выравниваются. И у вершины двойника концентрация напряжений практически соответствует величине напряжений при отсутствии внешнего воздействия (см. рис. 2). Таким образом, небольшая внешняя нагрузка приводит к существенному изменению полей напряжений у поверхности в области приложения усилия и незначительному изменению напряжений вдали от двойника. Происходит перераспределение напряжений с локализацией их на двойниковых границах у устья двойника. Действие внешней нагрузки ограничивается приповерхностной областью и практически не распространяется на глубину длины двойника. 


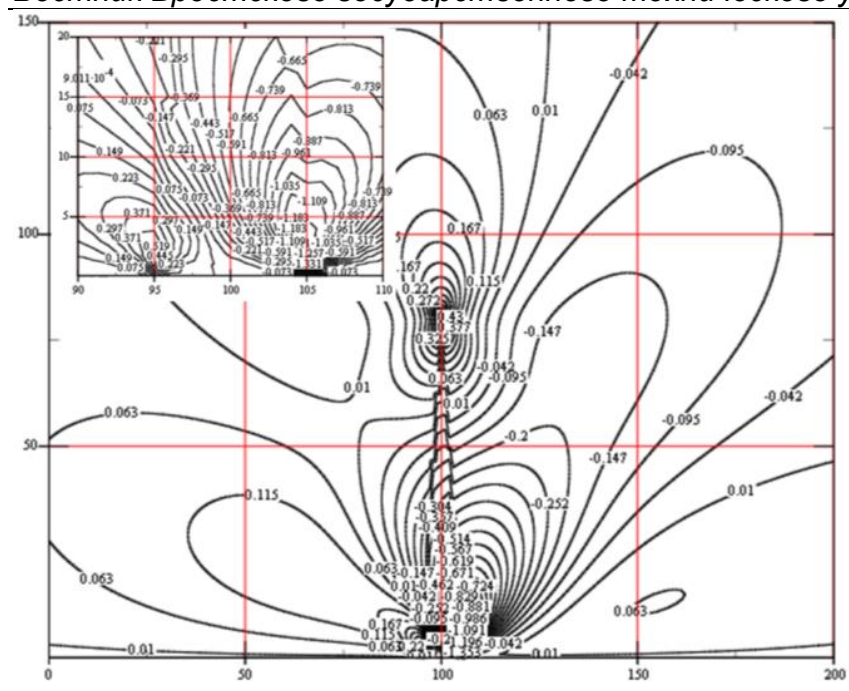

Рисунок 3 - Напряжения у клиновидного двойника при действии распределенной нагрузки $p(x)$ на поверхности в пределах ширины двойника

Целесообразно рассмотреть распределение напряжений у клиновидного двойника при той же эквивалентной внешней нагрузке в условиях неполного (частичного) контакта поверхностей. Силовое воздействие, распространяющееся, как и в предыдущем случае в пределах ширины двойника, т. е. $m=n=H / 2$, подчиняется закономерности (1) при $c=6$ (см. рис. 1, б). Безусловно имеем существенно несимметричную конфигурацию полей напряжений как на поверхности, так и вдоль двойниковых границ (рис. 4), причем с большей разницей численных значений вдоль границ по сравнению с равномерным нагружением. Так, на двойниковой границе у поверхности в зоне действия $p(s)=$ max напряжения увеличились практически в три раза, а на противоположной границе, где $p(s)=0$, уменьшились в два раза. И только в близлежащей к вершине двойника области наблюдается практически симметричное поле напряжений с такими же численными значениями, как и при равномерно распределенной внешней нагрузке. При этом следует также отметить соответствие полей в отношении знаков напряжений при плотном и частичном контакте поверхностей.

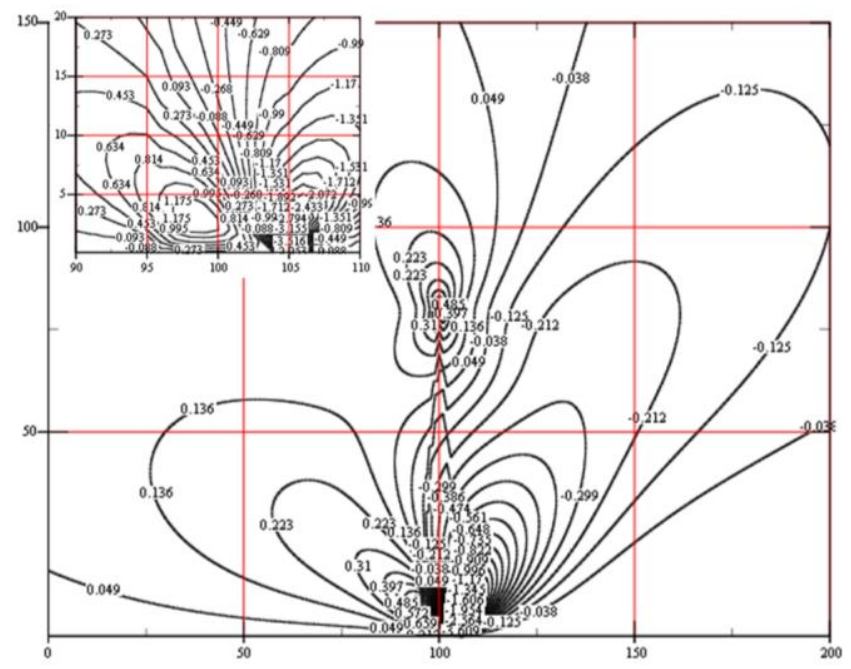

Рисунок 4 - Напряжения у клиновидного двойника при действии неравномерно распределенной нагрузки $\mathrm{p}(\mathrm{x})$ на поверхности в пределах ширины двойника

Представляет теоретический интерес и практическую целесообразность анализ распределения полей напряжений при увеличении площади контакта. На рис. 5 представлено поле напряжений у клиновидного двойника при действии равномерно распределенной нагрузки. Принимались границы силового воздействия $m=n=5 \mathrm{H}$, тогда эквивалентная нагрузка составит $p(s)=0,3 \mathrm{H} / \mathrm{M}$.

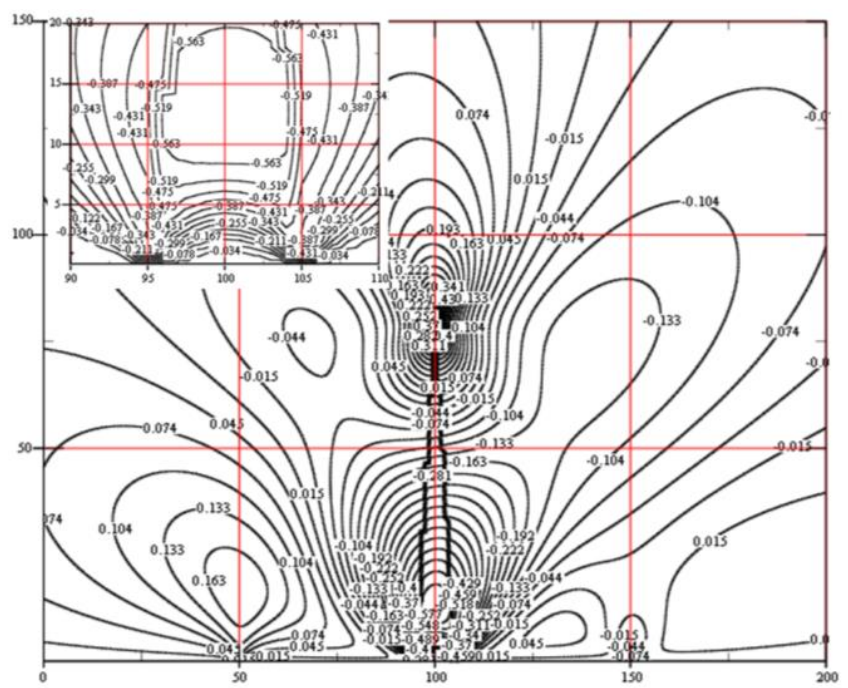

Рисунок 5 - Напряжения у клиновидного двойника при действии распределенной нагрузки $\mathrm{p}(\mathrm{x})$ на поверхности в интервале $10 \mathrm{H}$

Общая конфигурация поля напряжений в целом подобна расположению линий равных напряжений в материале без нагрузки (см. рис. 2). Это относится также и к численным значениям и последовательности изменения знаков напряжений. И только на граничных участках действия внешней нагрузки в приповерхностных областях наблюдается незначительная концентрация напряжений.

Аналогичную картину можно наблюдать при действии в том же интервале $m=n=5 \mathrm{H}$ неравномерно распределенной эквивалентной $(c=0,6)$ нагрузки (рис. 6). Отмечается только несколько большая несимметричность полей напряжений относительно плоскости двойникования, а также концентрация напряжений на границе $p(s)=$ max и некоторое увеличение в приповерхностной области на границе $p(s)=0$. Следовательно, при увеличении площади контакта действие внешней нагрузки незначительно влияет на напряженное состояние в области двойника и более значимо ее влияние на распределение напряжений в приповерхностной области материала.

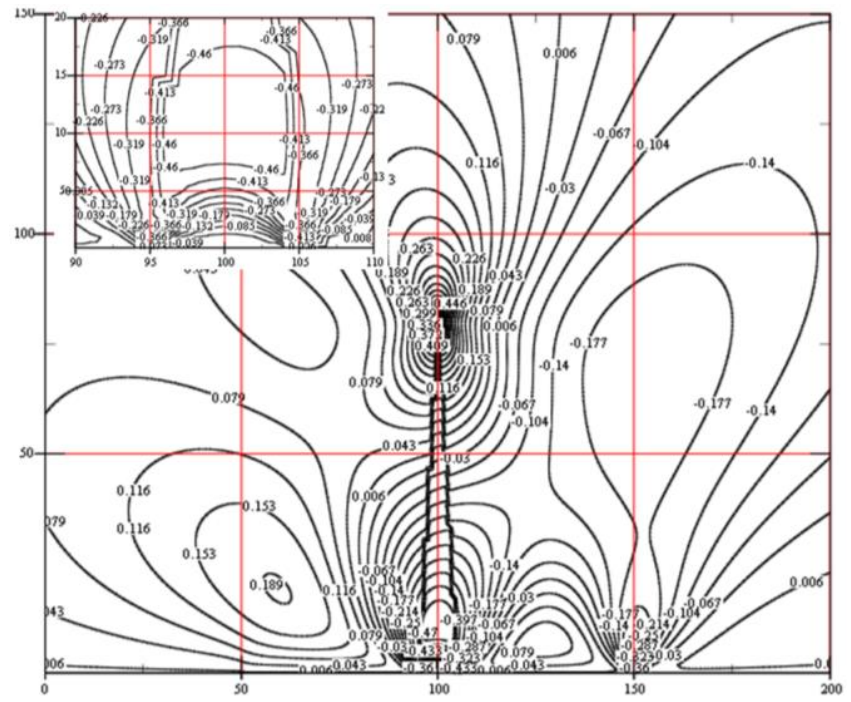

Рисунок 6 - Напряжения у клиновидного двойника при действии равномерно распределенной нагрузки $\mathrm{p}(\mathrm{x})$ на поверхности в интервале $10 \mathrm{H}$ 


\section{Заключение}

Таким образом установлено, что внешнее силовое воздействие заданной интенсивности незначительно влияет на концентрацию напряжений у вершины двойника. Более значимо его влияние на распределение полей напряжений в приповерхностной области материала. Аналогичное влияние наблюдается при увеличении зонь действия соответствующего поверхностного нагружения. Приложение неравномерной нагрузки увеличивает несимметричность полей напряжений относительно плоскости двойникования.

\section{Список цитированных источников}

1. Классен-Неклюдова, М. В. Механическое двойникование кристаллов / М. В. Классен-Неклюдова. - М. : Изд-во АН СССР, 1960. - 261 C.

2. Полухин, П. И. Физические основы пластической деформации / П. И. Полухин, С. С. Горелик, В. К. Воронцов. - М. : Металлургия, 1982. $-586 \mathrm{c}$.

3. Остриков, О. М. Механика двойникования твердых тел: монография / О. М. Остриков. - Гомель : ГГТУ им. П. О. Сухого, 2008. $301 \mathrm{c}$.

4. Федоров, В. А. Дислокационные механизмы разрушения двойникующихся металлов / В. А. Федоров, Ю. И. Тялин, В. А. Тялина. М. : Издательство Машиностроение-1, 2004. - 336 с.

5. Трефилов, В. И. Физические основы прочности тугоплавких металлов / В. И. Трефилов, Ю. В. Мильман, Ф. А. Фирстов. - К. Наук. думка, 1976. -315 с.

6. Финкель, В. М. Физические основы торможения разрушения / В. М. Финкель. - М. : Металлургия, 1977. - 360 с.

7. Остриков, О. М. Напряженное состояние у поверхности кристалла, деформируемой сосредоточенной нагрузкой, при наличии клиновидного двойника / О. М. Остриков // Журнал технической физики. - 2009. - Т. 79, № 5. - С.137-139.

8. Мышкин, М. К. Электрические контакты / Н. К. Мышкин, В. В. Кончиц, М. Браунович. - Долгопрудный: Издательский Дом «Интеллект», 2008. -560 c.

9. Остриков, О.М.Дислокационная макроскопическая модель клиновидного двойника / О. М. Остриков // Вестник ГГТУ им. П. О. Сухого. - 2006, № 2. - С. 10-18.

10. Косевич, А. М. О дислокационной модели двойникования / А. М. Косевич, А. А. Пастур // Физика твердого тела. - 1961. - Т.3, № 4. C. 1291-1297.

11. Остриков, О. М. Роль поверхности в формировании напряженного состояния у клиновидного нанодвойника / О. М. Остриков // Вестник ГГТУ им. П. О. Сухого. - 2012. - № 3. - С. 49-18.

12. Джонсон, К. Механика контактного взаимодействия / К. Джонсон. М.: Мир, 1989. -510 c.

13. Лившиц, Б. Г. Физические свойства металлов и сплавов / Б. Г. Лившиц. - М.: ГНТИ, 1959. - 386 с.

\section{References}

1. Klassen-Neklyudova, M. V. Mekhanicheskoe dvojnikovanie kristallov / M.V. Klassen-Neklyudova. - M.: Izd-vo AN SSSR, 1960. - 261 s.

2. Poluhin, P. I. Fizicheskie osnovy plasticheskoj deformacii / P. I. Poluhin, S. S. Gorelik, V. K. Voroncov. - M.: Metallur-giya,1982.- $586 \mathrm{~s}$.

3. Ostrikov, O. M. Mekhanika dvojnikovaniya tverdyh tel: mono-grafiya / O. M. Ostrikov. - Gomel': GGTU im. P. O. Suhogo, 2008. - $301 \mathrm{~s}$.

4. Fedorov, V. A. Dislokacionnye mekhanizmy razrusheniya dvojnikuyushchihsya metallov / V. A. Fedorov, YU. I. Tyalin, V. A. Tyalina. M. : Izdatel'stvo Mashinostroenie-1, 2004. - $336 \mathrm{~s}$.

5. Trefilov, V. I. Fizicheskie osnovy prochnosti tugoplavkih metallov / V. I. Trefilov, YU. V. Mil'man, F. A. Firstov. - K. : Nauk. dumka, 1976. $315 \mathrm{~s}$.

6. Finkel', V. M. Fizicheskie osnovy tormozheniya razrusheniya / V. M. Finkel'. - M.: Metallurgiya, 1977. - $360 \mathrm{~s}$.

7. Ostrikov, O. M. Napryazhennoe sostoyanie u poverhnosti kristalla, deformiruemoj sosredotochennoj nagruzkoj, pri nalichii klinovidnogo dvojnika/ O. M. Ostrikov // ZHurnal tekhnicheskoj fiziki. - 2009. T.79, №5. - S. 137-139.

8. Myshkin, M. K. Elektricheskie kontakty/ N. K. Myshkin, V. V. Konchic, M. Braunovich. - Dolgoprudnyj: Izdatel'skij Dom «Intellekt», 2008. $560 \mathrm{~s}$.

9. Ostrikov, O. M. Dislokacionnaya makroskopicheskaya model' klinovidnogo dvojnika / O. M. Ostrikov // Vestnik GGTU im. P. O. Suhogo. 2006, № 2. - S.10-18.

10. Kosevich, A. M. O dislokacionnoj modeli dvojnikovaniya / A. M. Kosevich, A. A. Pastur // Fizika tverdogo tela. - 1961. - T.3, №4. S. 1291-1297.

11. Ostrikov, O. M. Rol' poverhnosti $v$ formirovanii napryazhennogo sostoyaniya u klinovidnogo nanodvojnika / O. M. Ostrikov // Vestnik GGTU im. P.O. Suhogo. - 2012.- № 3.- S.49-18.

12. Dzhonson, K. Mekhanika kontaktnogo vzaimodejstviya / K. Dzhonson. - M.: Mir, 1989.- 510 s.

13. Livshic, B. G. Fizicheskie svojstva metallov i splavov / B. G. Livshic. M.: GNTI, 1959. - 386s

\section{Материал поступил в редакцию 03.05.2021}

